\title{
UCRL-CONF-206263
}

LAW RENCE LIVERMORE N A TIO NAL LABORATORY

\section{Simulation of Phase Effects in Imaging for Mesoscale NDE}

M. B. Aufderheide, III, A. Barty, H. E. Martz, Jr.

August 27, 2004

Review of Progress in Quantitative Nondestructive Evaluation Golden, CO, United States

July 26, 2004 through July 30, 2004 
This document was prepared as an account of work sponsored by an agency of the United States Government. Neither the United States Government nor the University of California nor any of their employees, makes any warranty, express or implied, or assumes any legal liability or responsibility for the accuracy, completeness, or usefulness of any information, apparatus, product, or process disclosed, or represents that its use would not infringe privately owned rights. Reference herein to any specific commercial product, process, or service by trade name, trademark, manufacturer, or otherwise, does not necessarily constitute or imply its endorsement, recommendation, or favoring by the United States Government or the University of California. The views and opinions of authors expressed herein do not necessarily state or reflect those of the United States Government or the University of California, and shall not be used for advertising or product endorsement purposes. 


\title{
SIMULATION OF PHASE EFFECTS IN IMAGING FOR MESOSCALE NDE
}

\author{
Maurice B. Aufderheide III, Anton Barty, and Harry E. Martz, Jr. \\ Lawrence Livermore National Laboratory
}

\begin{abstract}
High energy density experiments, such as those planned at the National Ignition Facility (NIF), use mesoscale targets with the goals of studying high energy density physics, inertial confinement fusion, and the support of national security needs. Mesoscale targets are typically several millimeters in size and have complex micrometer-sized structures composed of high-density metals and low-density foams and ices. These targets are designed with exacting tolerances that are difficult to achieve at present. Deviation from these tolerances can result in compromise of experimental goals and thus it is necessary to determine as-built properties of these targets using NDE techniques. Radiography and computed tomography are being used to investigate these targets, but the mix between phase and absorption information is difficult to separate, making interpretation of results difficult. We have recently improved the HADES radiographic simulation code to include phase in simulations, as an aid for doing NDE on mesoscale targets. In this paper we report on how we extended HADES to incorporate phase effects, and compare simulations with a variety of experimental test results.
\end{abstract}

\section{MOTIVATION}

The National Ignition Facility (NIF) is a Department of Energy facility currently under construction at Lawrence Livermore National Laboratory for the study of inertial confinement fusion and high energy density physics[1]. At this facility, 192 ultraviolet laser beams are focused to deliver $1.8 \mathrm{MJ}$ of energy and $500 \mathrm{TW}$ of power into millimeter-sized targets for a variety of implosion, hydrodynamic and radiation flow experiments. One application is the implosion of small DT capsules to achieve controlled nuclear fusion in the laboratory. Another application is the study of high energy density plasmas. In all of these applications, experimental results can have a sensitive dependence on instabilities[2], possibly seeded by imperfections in the targets.

The heart of these experiments is the target upon which the lasers are focused. These targets have sizes of several $\mathrm{mm}$ and are typically a series of spherical shells, with some 3D features. These shells can be a variety of metals, foams, exotic aerogels, and DT ice or liquid. Fabricating these features to the tolerances required for hydrodynamic stability is a challenging engineering problem with which LLNL is currently struggling. Typically the shells are assembled as hemi shells with step joints that may not be perfectly closed. Neighboring shells may not be in the direct contact requested in the specifications. Sphericity of shells, wall thickness, material uniformity and wicking of glue into foams are other issues that can affect the hydrodynamics of the as-built target.

Because of these issues, nondestructive evaluation of the final pre-shot assemblies is desperately needed. We call this effort "mesoscale NDE". An overview of these issues and the diagnostic probes under investigation are given in Figure 1. LLNL is looking at a variety of probes for various aspects of this problem[3]. Acoustics is being examined as probe of shell interfaces and step joint bonding. Nuclear magnetic resonance is being studied as a probe of foam integrity. Protons in the $\mathrm{MeV}$ range are being studied as a 
possible full assembly probe. We have been studying X-Ray phase contrast imaging as another full assembly probe.

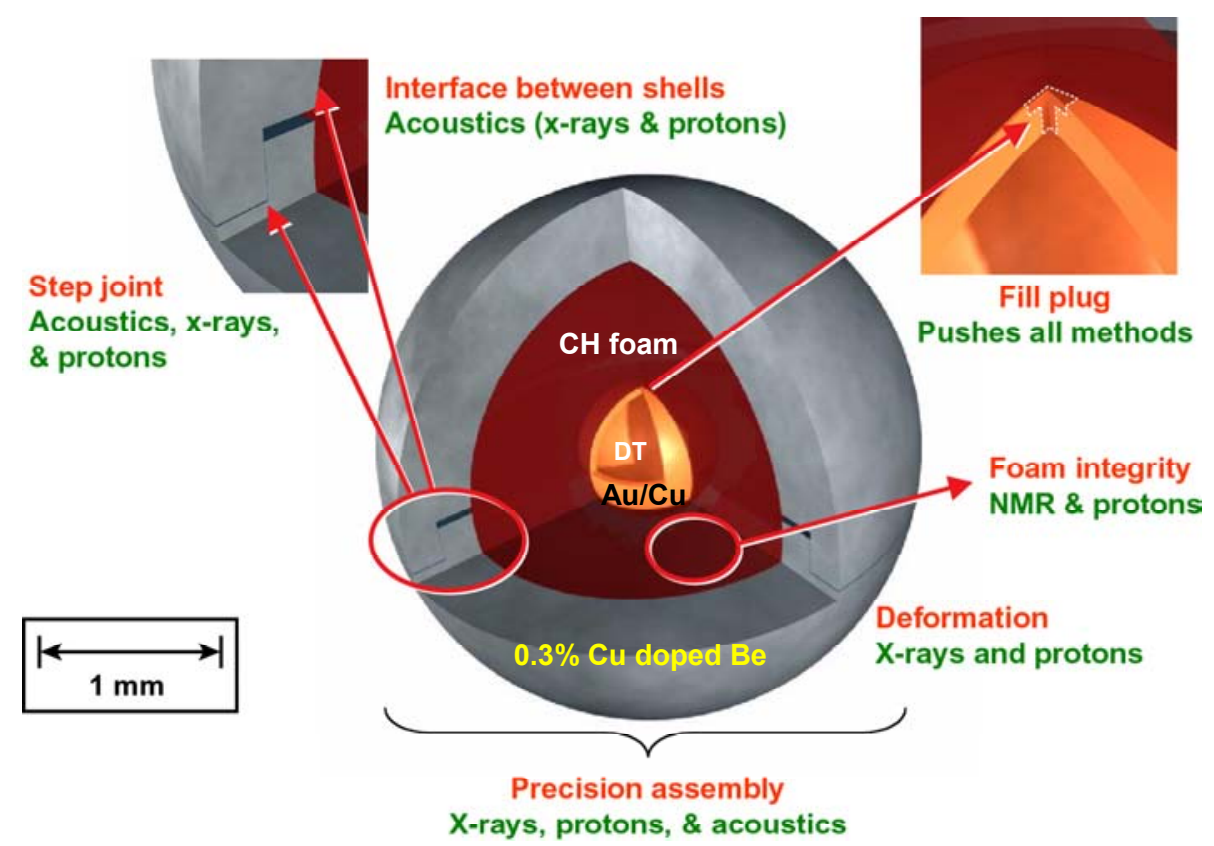

FIGURE 1: NIF target issues requiring NDE and diagnostic probes under investigation.

Because of the size of these objects, as well as the fact that we are trying to image low-density foams, liquids or ice inside metal shells, traditional absorption radiography is not advantageous. Energies higher than $100 \mathrm{keV}$ lead to low contrast images. In addition, traditional X-Ray sources have spot sizes that are too large. The size of these targets, and the materials that comprise them, lead us to microfocus or synchrotron sources in the 8 to $90 \mathrm{keV}$ energy range. When operating with these kinds of sources and the high resolution detectors required, we see not just absorption effects, but also phase effects such as refraction and diffraction in the radiographic images. These effects can help to enhance low-density features, such as deuterium/tritium (DT) vapor/ice interfaces inside a metal shell, as well as interfaces. However these benefits are compromised by the fact the basic assumptions for tomography[4] are violated and tomographic reconstructions are compromised.

In this paper we describe how we are developing tools to model this kind of radiography in the HADES radiographic simulation code[5]. We begin by discussing XRay phase effects. We describe how these effects are incorporated in HADES and show some examples. We finish with a discussion of future work.

\section{X-RAY PHASE EFFECTS}

For microfocus sources or synchrotron X Ray sources, the source "size" is small enough that the radiation has sufficient spatial coherence for phase contrast effects to be observed. In addition to spatial coherence it is necessary to have high detector resolution, as well as adequate propagation distance downstream of the object, in order to render visible the phase structure of the object. Many physicists automatically connect the notion of phase measurement with techniques involving interferometry. However, it has long been known that propagation of an optical field through free space can render the 
phase structure of an object visible in the measured intensity distribution[6]. Although of a shorter wavelength than visible light, X-rays obey fundamentally the same wave equations as visible light and, at high resolution, the wave properties of the radiation cannot be ignored. In particular, the use of a microfocus source and free space propagation provides a simple but powerful method for X-ray microscopy, and is one in which the phase effects of the object can not be ignored.[7,8].

The effect of phase on the detected image can be thought of by analogy with normal visible light. For slowly varying structures, the object phase acts like a lens refracting the $\mathrm{X}$-rays, redirecting the energy flow and forming contrast in a downstream image in much the same way that ripples on a swimming pool form bright and dark lines on the pool floor. For more sharply varying structures, such as the sharp phase gradients present at tangents to a sphere, the situation is analogous to the diffraction of laser light past a sharp barrier.

This phase sensitive approach to radiography exploits Fresnel diffraction and has been called "phase-contrast" imaging (although, as noted by Fitzgerald[9], this approach is distinct from optical phase-contrast imaging). This type of radiography was first explored by a number of researchers in the mid 1990s[10,11,12,13,14,15]. This approach is very similar to Gabor's original concept of inline holography[16].

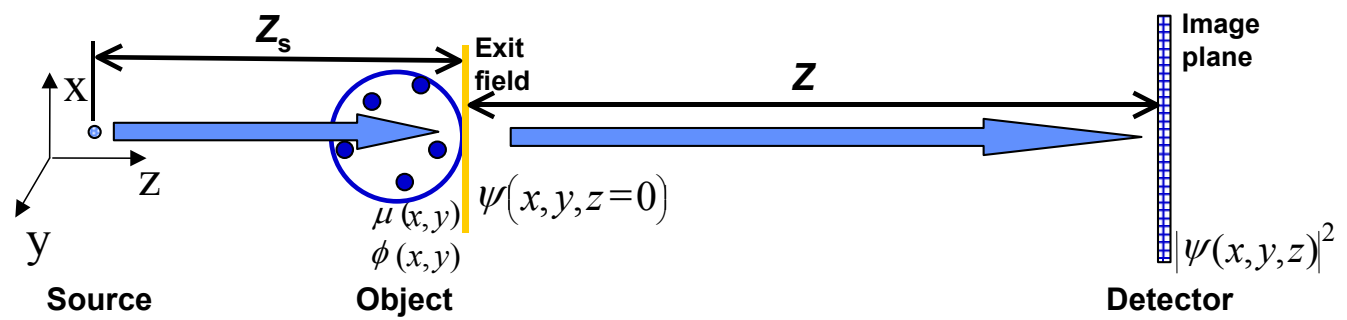

FIGURE 2: Geometry of phase sensitive radiography. Coherent $\mathrm{X}$ Rays are generated by the source, pass through the object and emerge as the exit field $\psi(\mathrm{x}, \mathrm{y}, \mathrm{z}=0)$. The field then propagates to $z$ and the intensity is given by $|\psi(\mathrm{x}, \mathrm{y}, \mathrm{z})|^{2}$.

The geometry for phase sensitive radiography is given in Figure 2. The source is a distance $z_{s}$ from the exit plane of the object. This plane is a distance $z$ from the detector. At the exit plane of the object, the X-Ray field emerges and begins propagation through the "vacuum" to the image plane. At this plane, the detector measures the intensity, which is the square of the wave function.

One can think of the object as a lens or diffracting object through and around which the wave propagates. In such a case, it is possible to compute the downstream amplitude using the Fresnel integral:

$$
\Psi(x, y, z)=\frac{i}{\lambda z} e^{-i k z} \iint \Psi\left(x^{\prime}, y^{\prime}, 0\right) e^{\frac{i \pi}{\lambda z^{\prime}}\left[\left(x-x^{\prime}\right)^{2}+\left(y-y^{\prime}\right)^{2}\right]} d x^{\prime} d y^{\prime}
$$

where $z^{\prime}$ is given by $z /\left(1+z / z_{s}\right)$. This integral can be simplified by using the convolution form of the Fresnel Integral:

$$
\Psi(x, y, z)=-e^{i k z} \mathfrak{J}^{-1}\left\{e^{-i \pi \lambda z^{i i^{2}+j j^{2}}} \frac{\ln ^{2}}{(N \Delta x)^{2}} \mathfrak{I}\left\{\Psi\left(x^{\prime}, y^{\prime}, 0\right)\right\}\right\}
$$

where $\mathfrak{I}$ and $\mathfrak{I}^{-1}$ are the Fourier and inverse Fourier Transform respectively, $N$ is the number of pixels in the image, $\Delta x$ is the size of each pixel, and $i i$ and $j j$ are the image indices. This form is useful because the wave function can be treated as a complex image and is computed quickly using fast Fourier transforms. 


\section{INCORPORATING PHASE EFFCTS IN HADES}

In order to apply these techniques, it is necessary to compute $\psi(x, y, z=0)$. This quantity is estimated using the HADES ray tracing code. The wave function at the exit plane is given by

$$
\psi(x, y, z=0)=\sqrt{e^{-\mu(x, y)}} e^{i \phi(x, y)}
$$

where $\mu(x, y)$ is the image of the absorption path length through the object and $\phi(x, y)$ is the image of the phase path length through the object. Both the absorption and phase through the objects are computed within HADES using ray tracing techniques.

The index of refraction, $n$, for materials being traversed by X-Rays is a complex number given by $n=1-\delta-i \beta$, where $\delta$ is the refractive index and $\beta$ is the absorption index. These indices are computed from the atomic scattering factors $f_{1}$ and $f_{2}$ as follows:

$$
\begin{aligned}
& \delta=\frac{r_{e} \lambda^{2}}{2 \pi} \rho N_{A} \sum_{i} \frac{X_{i}}{A_{i}} f_{1, i} \\
& \beta=\frac{r_{e} \lambda^{2}}{2 \pi} \rho N_{A} \sum_{i} \frac{X_{i}}{A_{i}} f_{2, i}
\end{aligned}
$$

where $r_{\mathrm{e}}$ is the classical electron radius, $\lambda$ is the $\mathrm{X}$-Ray wave length, $\mathrm{N}_{\mathrm{A}}$ is Avogadro's number, $X_{i}$ is the mass fraction of the $i^{\text {th }}$ element, $A_{i}$ is the atomic mass of the $i^{\text {th }}$ element, $f_{1, i}$ and $f_{2, i}$ are the first and second atomic scattering factors of the $i^{\text {th }}$ element, $\rho$ is the density of the compound, and the summation is over all $i$ isotopes in the compound. The atomic scattering factor tables were obtained from the LBNL Center for X-Ray Optics website [17], which are modified tables based on the work of Henke et al.[18]. In our work, we have treated each compound as a mixture of isotopes and all chemical and molecular effects have been ignored. In the keV energy range we are studying, this is a fine approximation.

From these scattering factors, we can compute the absorption and phase path lengths, $\mu$ and $\phi$, through a zone using

$$
\begin{aligned}
& \mu=\frac{4 \pi}{\lambda} \beta \Delta \ell \\
& \varphi=\frac{2 \pi}{\lambda} \delta \Delta \ell
\end{aligned}
$$

where $\Delta \ell$ is the length of the track through the zone. HADES uses these expressions and tables to compute the images $\mu(x, y)$ and $\phi(x, y)$. These images are then combined as in Equation (3) to obtain the X-Ray exit field. In a post processing step, the exit field is propagated downstream to the detector plane using the convolution form of Fresnel's integral given in Equation (2). For the Fourier transforms, we use the FFTW library[19].

In our current phase contrast implementation within HADES, the user specifies the object, $z_{s}, z$, and the X-Ray energy. HADES then computes $f_{1, i}$ and $f_{2, i}$ for this energy and material and computes $\mu(x, y)$ and $\phi(x, y)$ through the specified object and finally the intensity at the image plane. Blurring due to source size and detector resolution is done after the simulation. Detector blur and quantum efficiency tables, regularly used in the phaseless implementation of HADES, are not currently enabled in this part of HADES. Polychromaticity and finite source size also are not yet enabled in the phase contrast implementation of HADES. These improvements will be added to HADES in time.

There is another possible issue in modeling phase contrast physics with HADES. In computing the exit wave of the X-Ray field, we are using HADES to ray trace through 
the object and acting as if the object were very thin relative to the diffraction physics occurring in the problem. However, it is possible that diffraction from features within the object could occur, leading to a more complex exit wave than is being approximated in this treatment by HADES. In such a case, the object would need to be broken into slices perpendicular to the radiographic axis and the X-Ray field would have to be computed and propagated at these intermediate steps. This is called a multi-slice approximation[20] and the general condition[21] when multi-slice is necessary is given by

$$
O_{z}>\frac{f^{2}}{\lambda}
$$

for a feature size $f, \mathrm{X}$-Ray wavelength $\lambda$ and object size along the radiographic axis $O_{z}$. For $1 \mu \mathrm{m}$ features and $8 \mathrm{keV} \mathrm{X}$-Rays, objects greater that roughly $6 \mathrm{~mm}$ in size would need a multi-slice treatment; this is close to the NIF targets of interest. For $30 \mathrm{keV} \mathrm{X}$ Rays, the object size limit grows to $2.4 \mathrm{~cm}$, which is larger than NIF target sizes.

Preliminary detailed wave propagation studies have examined these cases and have found that the ray-tracing technique is adequate for the targets and energies of interest here. We could add a multi-slice treatment to HADES (at the cost of greater computational complexity), but will not for now, since it does not seem to be necessary.

\section{RESULTS}

As an example of HADES' new capability, we have computed radiographs with phase effects for a $\mathrm{Si}_{3} \mathrm{Ni}_{4}$ mandrel with $925 \mu \mathrm{m}$ radius surrounded by a $75 \mu \mathrm{m}$ thick diamond shell (DS). We simulate radiographs for $8 \mathrm{keV}$ and $30 \mathrm{keV} \mathrm{X-Rays} \mathrm{with} z_{s}$ set to infinity (a parallel beam) and $z$ set to $10 \mathrm{~cm}$. In Figure 3, we show a radial profile of the simulation. We have used $1 \mu \mathrm{m}$ pixels for the images. Three cases are displayed for each energy. First, we display the absorption only case. Second, we display the case with phase effects included. Third, we display the phase effects with an experimentally relevant resolution applied.

It can be seen that the $30 \mathrm{keV}$ absorption only case has little dynamic range over the region of the diamond shell. $8 \mathrm{keV}$ is a more appropriate energy for this object. Many high frequency oscillations of relatively small amplitude can be seen in each case when phase effects are included. Both energy cases show large oscillations in intensity near the $\mathrm{Si}_{3} \mathrm{Ni}_{4} / \mathrm{DS}$ and $\mathrm{DS} /$ Vacuum interfaces. Diffraction off these interfaces is resulting in very vigorous constructive and destructive interference near these interface locations. At $8 \mathrm{keV}$, a great deal of destructive interference occurs near both interfaces, making dimensional estimates difficult.

The high frequency oscillations seen

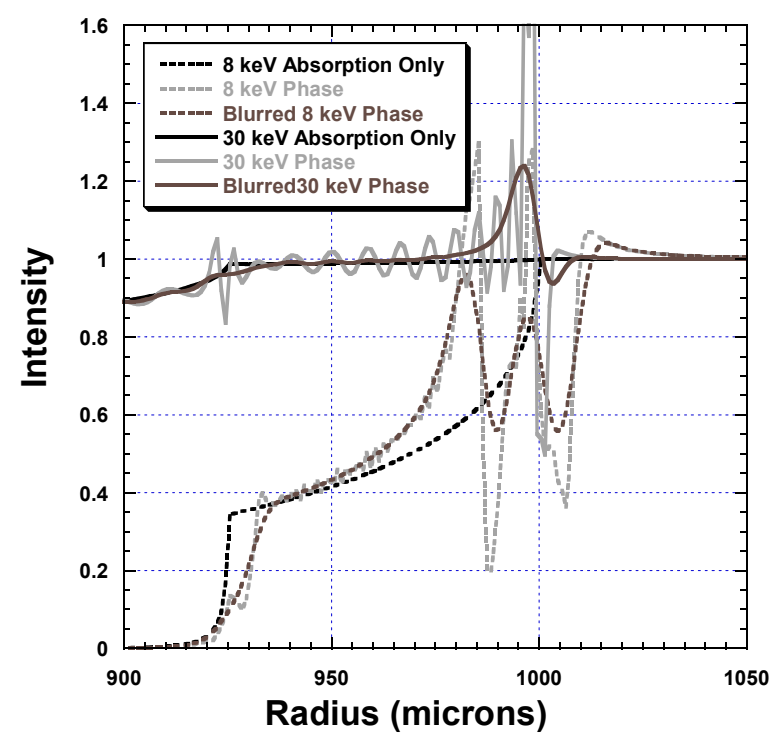

FIGURE 3: 8 and $30 \mathrm{keV}$ simulated radiographic profiles through a $\mathrm{Si}_{3} \mathrm{~N}_{4}$ mandrel enclosed in a 75 $\mu \mathrm{m}$ thick diamond shell. in the simulations with phase effects are never seen experimentally. Detectors have finite 
spatial resolution and tend to blur out the signal. For the third simulation of each case, we have added an experimentally appropriate Gaussian blur of $\sigma=3 \mu \mathrm{m}$. This blurring smoothes out the high frequency oscillations, but does not remove the large oscillations at the interfaces.

If one compares the smoothed phase profiles with the no phase profiles, several features are observed. Firstly, a significant amount of intensity has been moved to different radii. In these cases, diffraction through the object has lead to this intensity being scattered about the interfaces. Secondly, interference effects have compromised the profiles so badly that they are hard to interpret from a radiographic or tomographic standpoint. Tomography assumes that only direct radiation is reaching each pixel. Here, the phase effects have scattered the direct radiation in highly correlated ways. If one were to tomographically reconstruct these radiographs, the resulting objects would be very equivocal. Shell densities would be incorrect and interfaces would be difficult to identify. One of the reasons for implementing this capability in HADES is to see how we can get back to data that can be reconstructed in a reliable fashion.

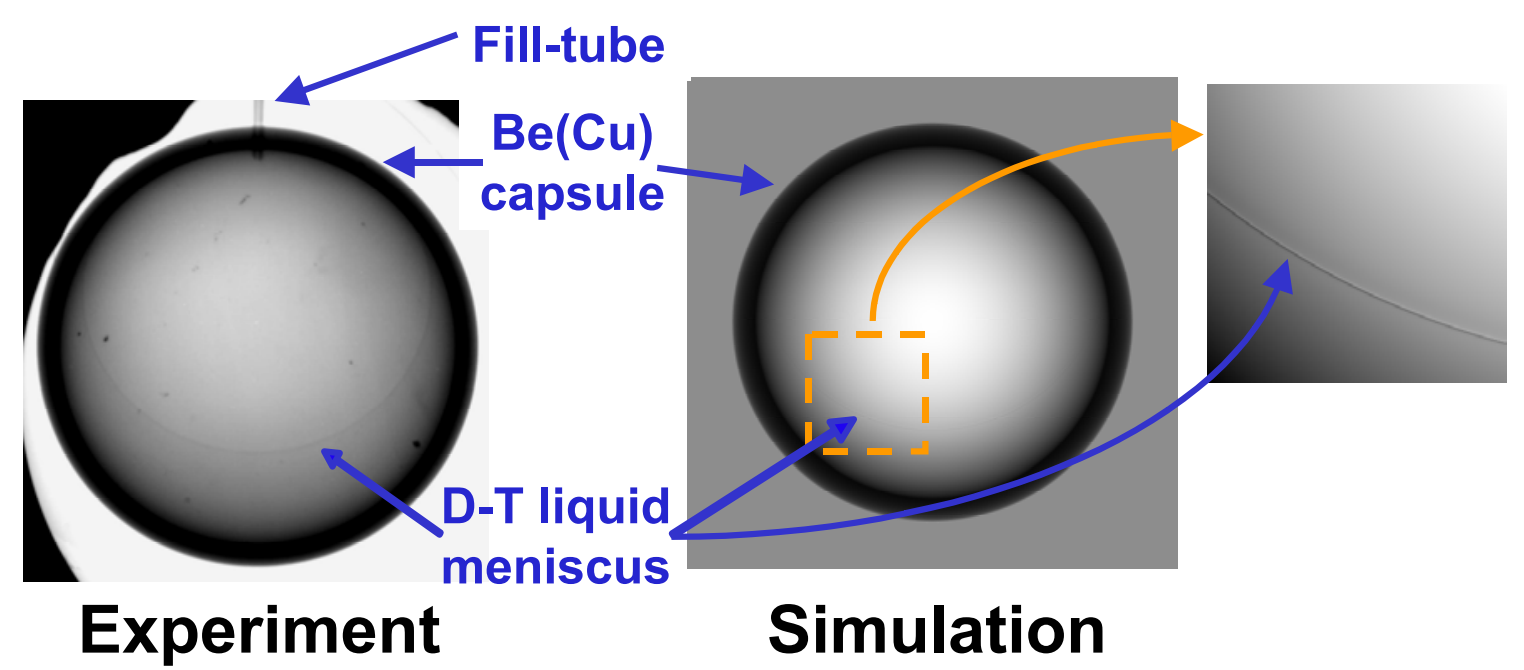

FIGURE 4: Phase radiography of a NIF target, successfully imaging the DT liquid meniscus. The experimental image (left); a HADES simulation (right). Only phase effects can image this meniscus.

In Figure 4 we display an experimental radiograph generated with LLNL's KCAT radiographic system[22] of a simple NIF fusion target. For this experiment, the KCAT system used a microfocus X-Ray tube with a peak voltage of $70 \mathrm{keV}$ and a scintillator optically coupled to a CCD camera. The target was a $115 \mu \mathrm{m}$ thick shell of Be doped with $1 \% \mathrm{Cu}$ by weight and $1 \mathrm{~mm}$ radius filled with liquid DT. In the experimental image the meniscus of the DT liquid is visible even inside the metal shell. The density of the DT liquid is $0.1 \mathrm{~g} / \mathrm{cc}$, while the density of the shell is $1.85 \mathrm{~g} / \mathrm{cc}$. With normal transmission radiography, the transition from DT vapor to DT liquid is so obscured by the metal shell that it is not visible. The only reason that the DT meniscus is visible is because phase effects enhance the DT layer.

We also show a HADES simulation of this system in Figure 4. For the simulation we have used $10 \mathrm{keV}$ as the X-Ray energy and have displayed intensity, unblurred and unmodulated by detector response. It can be seen that the simulation does not match the dynamic range of the experimental radiograph. Also, the DT vapor/liquid interface is not as visible in the simulation as in the experimental image. It is likely that the source of these disagreements is the relative simplicity of the simulation. In future work we will include the spectral distribution of the source and the detector response. 


\section{CONCLUSIONS AND FUTURE WORK}

In this paper we have described how the National Ignition Facility needs mm-sized targets for its experiments in inertial confinement fusion and high energy density physics. This is leading to the development of NDE tools for objects in this size range. One of the techniques we are studying is phase enhanced radiography. This study is possible because of the availability of microfocus sources and synchrotron X-Ray sources, which have the spatial coherence to prevent the phase effects being washed out.

Because of this work in phase enhanced radiography, we have been upgrading our radiographic simulation code, HADES, to treat this kind of Physics. We have imported the relevant cross sections and have implemented Fresnel integration to model the downstream propagation of the X-Ray exit wave. We have just begun to validate the code against data. There is still much work to do to improve the realism of the simulation, but we are making progress.

We are currently working on validating the code against a variety of images taken with a variety of spatially coherent sources. We are already using the LLNL KCAT system, as mentioned in the previous section. A number of LLNL collaborators have time on the LBNL Advanced Light Source[23] and are generating validation data sets for us. LLNL has recently purchased an Xradia $\mu \mathrm{XCT}$ system[24] for doing microtomography and radiography.

We will be looking at a variety of objects in order to validate this new HADES modeling area. Fully absorbing straight edges made of $\mathrm{Au}$ or Ta will be used to study diffraction effects and blur functions. Plastic rods will be used to study the interplay between absorption and phase. Some phantoms will be used to study the effects of innerobject diffraction. We also have cylindrical and spherical reference standards that have features designed to be reminiscent of NIF targets[25]. We will be radiographing these objects as validation tests for HADES. In addition, we will use semi-analytic diffraction cases for verifying HADES algorithms.

\section{ACKNOWLEDGMENTS}

We thank Alexis Schach von Wittenau, Bernie Kozioziemski, Sean Lehman, and Dan Schneberk for useful discussions. Some of this work has been funded in part by a Laboratory Directed Research and Development exploratory research grant provided by the Engineering Directorate. This work was performed under the auspices of the U.S Department of Energy by the University of California, Lawrence Livermore National Laboratory under Contract No. W-7405-Eng-48.

\section{REFERENCES}

1. NIF website: http://www.llnl.gov/nif

2. Lindl, J.D., Intertial Confinement Fusion, AIP Press, (1998), particularly Chapters 6 and 9.

3. Martz Jr., H. E., and G. F. Albrecht, "Nondestructive Characterization Technologies for Metrology of Micro/Mesoscale Assemblies," Proceedings of: Machines and Processes for Micro-scale and Meso-scale Fabrication, Metrology, and Assembly, ASPE 2003 Winter Topical Meeting, Gainesville, FL, (2003), 131141.

4. Kak, A.C., Slaney, M., Principles of Computerized Tomographic Imaging, IEEE Press, 1987. 
5. Aufderheide, M. B., Slone, D. M. and Schach von Wittenau, A. E., "HADES, a radiographic simulation code", in Review of Progress in Quantitative Nondestructive Evaluation, edited by D. O. Thompson and D. E. Chimenti, AIP Conf. Proc. \#557, Vol. 20A (2000), pp. 507-513.

6. Zernike, E., "Phase contrast: a new method for the microscopic observation of transparent objects", Physica, 9, 686-693 (1942).

7. Pogany et.al.. "Contrast and resolution in imaging with a microfocus x-ray source", Rev.Sci.Instrum., 68, 2774-2782 (1997).

8. Gureyev et.al., "On X-ray phase imaging with a point source”, J.Opt.Soc.Am., A15, 579-585 (1998).

9. Fitzgerald, R., "Phase Sensitive X-Ray Imaging", Physics Today, 53, 23-26 (2000).

10. White, V., and Cerrina, F., "Metal-less X-Ray phase shift masks for nonlithography", J. Vac. Sci. Technol. B10, 3141-3144 (1992).

11. Snigirev, A., et al., "On the possibilities of X-Ray phase contrast microimaging by coherent highenergy synchrotron radiation”, Rev. Sci. Instrum., 66, 5486-5492 (1995).

12. Cloetens, P., et al., "Phase objects in synchrotron radiation hard X-Ray imaging", J. Phys. D: App. Phys., 29, 133-146 (1996).

13. Cloetens, P., et al., "Observation of microstructure and damage in materials by phase sensitive radiography and tomography”, J. Aplp. Phys., 81, 5878-5886 (1997).

14. Nugent, K.A., et al., "Quantitative phase imaging using hard X-Rays", Phys. Rev. Lett., 77, 29612964 (1996).

15. Wilkins, S.W., et al., "Phase-contrast imaging using polychromatic hard X-Rays", Nature, 384, 335338 (1996).

16. Gabor, D., “A new microscopic principle”, Nature, 161, 777-778 (1948).

17. Web address: http://www.cxro.lbl.gov/optical_constants/asf.html

18. Henke, B.L., Gullikson, E.M., and Davis, J.C., "X-ray interactions: photoabsorption, scattering, transmission, and reflection at E=50-30000 eV, Z=1-92", Atomic Data and Nuclear Data Tables 54 no.2, 181-342 (July 1993).

19. Frigo, M., and Johnson, S.G., "FFTW: An Adaptive Software Architecture for the FFT", Proc. ICASSP 1998, 3, 1381-1384 (1998). See also the FFTW website: http://www.fftw.org.

20. Hare, A.R., and Morrison, G.R., "Near-field soft X-Ray diffraction modeled by the multislice method", J. Mod. Opt., 41, 31-48 (1994).

21. Cowley, J.M., Diffraction Physics $2^{\text {nd }}$ Edition, North-Holland, Section 10.6.

22. Waters, A. M. (2001), "Three-dimensional analysis of voids in AM60B magnesium tensile bars using computed tomography imagery," Ph.D. dissertation, Johns Hopkins University, Baltimore, MD.

23. Website is http://www-als.lbl.gov/als/

24. Website is http://www.xradia.com/

25. Waters, A., H. Martz, C. Logan, J. Gross and D. Chinn, "KCAT Performance Summary - Update", Lawrence Livermore National Laboratory, Livermore, CA, UCRL-TR-203519 (2004). 